Meta

Journal des traducteurs

Translators' Journal

\title{
L’interprétation judiciaire des textes législatifs bilingues
}

\section{Marie Lajoie}

Volume 24, numéro 1, mars 1979

La traduction juridique

URI : https://id.erudit.org/iderudit/003376ar

DOI : https://doi.org/10.7202/003376ar

Aller au sommaire du numéro

Éditeur(s)

Les Presses de l'Université de Montréal

ISSN

0026-0452 (imprimé)

1492-1421 (numérique)

Découvrir la revue

Citer cet article

Lajoie, M. (1979). L’interprétation judiciaire des textes législatifs bilingues.

Meta, 24(1), 115-124. https://doi.org/10.7202/003376ar d'utilisation que vous pouvez consulter en ligne.

https://apropos.erudit.org/fr/usagers/politique-dutilisation/ 


\section{L'interprétation judiciaire des textes législatifs bilingues}

Il serait difficile de parler de l'interprétation des textes législatifs bilingues sans parler de la rédaction et de la traduction de ces textes. Outre une interaction commune, chacune de ces deux disciplines comporte ses caractéristiques et ses problèmes propres qu'il serait trop long de vouloir exposer ici. Surtout si l'on considère que ces caractéristiques et problèmes diffèrent selon que l'on s'attache à les cerner au niveau de la législation fédérale ou au niveau de la législation provinciale et tout particulièrement québécoise. Nous nous limiterons donc aux aspects de la rédaction et de la traduction les plus intimement liés à l'interprétation judiciaire.

C'est la rédaction des textes qui appelle l'interprétation des tribunaux. C'est en effet à l'interprétation que l'on évalue principalement la qualité d'un texte législatif. Au Canada les recueils de jurisprudence comportent, tant en anglais qu'en français, un grand nombre de décisions cherchant à définir des expressions ou des mots dont le sens est en général courant. Il y a recherche du sens des mots ou des concepts afin de savoir de quelle façon il convient de les appliquer à un contexte précis. À cette fin, le juge recherche d'abord et avant tout l'intention du législateur ; c'est la première règle d'interprétation.

Définissons pour nos besoins trois types d'interprétation. D'abord l'interprétation unilingue, lorsque les deux versions d'un même texte concordent; en second lieu l'interprétation bilingue, lorsque les deux versions concordent aussi, mais où l'une ajoute une certaine précision à l'autre ; et enfin l'interprétation bilingue, elle aussi, lorsque les deux versions d'un même texte divergent.

\section{L'INTERPRÉTATION UNILINGUE}

Les exemples du premier type sont en nombre infiniment supérieur à ceux des deux autres. Ainsi, l'arrêt Sa Majesté la Reine c. Monique McCutcheon ${ }^{1}$ constitue un exemple d'interprétation assez courant dans lequel la traduction ne joue pour ainsi dire aucun rôle, vu l'équivalence des deux versions. Le problème découle ici de l'ambiguïté des mots de l'article 170 du Code criminel. La Cour d'appel du Québec tente de déterminer si, comme l'affirme Sa Majesté la Reine, l'effeuilleuse intimée était bien nue ou encore vêtue de façon à offenser

1. R. c. Monique McCutcheon, (1977) C.A., 103 (résumé). 
la décence ou l'ordre public. Il est en effet à tout le moins déroutant de lire à cet article que l'on peut être nu tout en étant vêtu. Le juge Owen résume bien l'ironie de la situation :

Sec. 170 Cr. C. provides in subsection (1) that everyone who is nude in a public place is guilty of an offence. Up to that point the wording is clear. Nude with respect to a person has a generally accepted meaning. It means naked, bare, without covering, unclothed. However subsection (2) of the section provides that for the purpose of Sec. $170 \mathrm{Cr}$. C. a person is nude who is so clad as to offend against public decency or order. This is confused and confusing. In ordinary language a person who is nude is unclad and a person who is clad is not nude, but this subsection (2) provides that for the purposes of Sec. $170 \mathrm{Cr}$. C. a person is nude who is clad. I do not think it should be said that Section $170 \mathrm{Cr}$. C. is clear and unambiguous - particularly in view of the fact that so many different judges of different courts have disagreed as to its meaning ${ }^{2}$.

A la lecture d'un texte, l'intention du législateur demeure souvent obscure. Cet article 170, rédigé à la mode anglo-saxonne de common law, illustre la possibilité qu'a le législateur de donner aux mots les plus usuels (ici nude - nu) le sens qu'il entend leur donner. Encore faut-il qu'il se fasse comprendre. Il peut à loisir restreindre, étendre ou modifier totalement le sens des mots. C'est ainsi que le Game and Fish Act de l'Ontario fait d'une arbalète une arme à feu :

\&I. In this Act,

$\dddot{8}$. Fire-arm $\gg$ includes an air or pellet gun and a longbow and a crossbow ;

... ${ }^{8}$ s.

Les exemples de ce genre sont nombreux ; nous avons pourtant vu ici le genre de réflexe que ce mode de rédaction entraîne même chez le lecteur le plus averti.

\section{L'INTERPRETATION BILINGUE,}

LORSQUE LES DEUX VERSIONS CONCORDENT

Vient ensuite l'interprétation bilingue où deux versions concourent à la précision du sens. Il arrive parfois que le traducteur vienne au secours du rédacteur en l'aidant à préciser sa pensée ou en excluant le doute quant au sens des mots. Ainsi, dans $S t$-Germain c. $R$., le juge Lajoie de la Cour d'appel s'attache à définir les termes « Loi \$ — Act » et « loi » — «law ».

Lorsque le Législateur réfère au Code criminel, il y emploie les mots « la présente loi », « this Act ». Les termes « Loi», «Act», désignent une loi...

Lorsque le Législateur fédéral emploie les mots « la loi », \& law », comme à l'article 202(2) C. Cr., ou encore aux articles 25(1) ou 26 C. Cr., il leur donne un sens beaucoup plus large que celui d'une loi. La loi, «law », c'est l'ensemble des dispositions législatives adoptées par quelqu'autorité compétente que ce soit et qui régissent la vie en société ".

2. Sa Majesté la Reine c. Monique McCutcheon, 1977, C.A. Montréal, no 10-000279-750, le juge Owen, à la p. 8. (Le souligné est mien.)

3. The Game and Fish Act, R.S.O., 1970, c. 186, a. 1 .

4. Jacques St-Germain c. $R$., (1976) C.A. 185, aux pages 190-191. 
Dans ce cas, la présence de deux langues n'a certes pas nui à l'éclaircissement du contexte, bien au contraire. L'appelant soutenait en effet que le devoir légal imposé par la loi à l'article 202 du Code criminel signifiait un devoir imposé par la Loi criminelle fédérale. Dans ce type d'interprétation comme dans le suivant, le fait d'être bilingue apporte au juge un élément de précision non négligeable.

\section{L'TNTERPRÉTATION BILINGUE, \\ LORSQUE LES DEUX VERSIONS DIVERGENT}

Vient enfin l'interprétation encore une fois bilingue, mais où les deux versions officielles d'un même texte législatif divergent. Ce phénomène demeure relativement fréquent. Les exemples en sont principalement concentrés au Québec ainsi qu'à la Cour suprême et à la Cour fédérale. Ce type d'interprétation survient lorsque à des problèmes de rédaction s'ajoutent des problèmes de traduction.

Dans une toute récente décision de la Cour d'appel du Québec, le juge Antonio Lamer expose certaines règles qui s'imposent à un juge devant la nonconcordance des deux versions d'une disposition législative. Les trois juges qui composent le banc sont d'accord, dans $B$. P. Pétroles Limitée c. Sa Majesté la Reine, que les versions anglaise et française de l'article 11 , alinéas $\mathrm{g}$ ) et $\mathrm{h}$ ) de la Loi sur le dimanches divergent. Le juge Lamer explique sa pensée en ces termes :

Il existe une divergence dans le choix des termes par le législateur selon qu'il s'agit de la version anglaise ou française de l'article 11 de la Loi sur le dimanche.

Il s'agit donc pour nous d'appliquer la disposition de l'alinéa d) du paragraphe (2) de l'article 8 de la Loi sur les langues officielles qui se lit comme suit :

d) s'il y a, entre les deux versions du texte législatif, une différence autre que celle mentionnée à l'alinéa c), on donnera la préférence à la version qui, selon l'esprit, l'intention et le sens véritables du texte, assure le mieux la réalisation de ses objets.

$1968-69$, c. 54 , art. 8 .

On doit, je crois, rechercher d'abord les objets que le législateur voulait réaliser par sa loi par l'application des règles générales d'interprétation des lois pour ensuite, et seulement lorsque la divergence des deux textes est irréductible, appliquer la règle de l'article 8 et donner la préférence à la version qui en assurera le mieux la réalisation. Ceci pour dire que c'est d'abord dans son texte que l'on doit chercher l'objet d'une loi en donnant aux mots qu'il emploie (en l'absence de définitions spéciales) leur sens ordinaire.

Le texte de la loi est tout autant français qu'anglais et l'article $8(2)$ d) ne défend pas dans la recherche de l'intention du législateur (i.e. les objets du texte législatif) d'interpréter les termes d'une version par ceux qu'employait le législateur dans l'autre.

S. Loi sur le dimanche, S.R.C., 1970, c.L-13. 
A première vue on croit retrouver une solution à même le texte des alinéas g) et h) de l'article 11 de la Loi sur le dimanche. En effet, le concept rendu par l'expression « and work incidental thereto » employé aux alinéas g) et $h$ ) de l'article 11 est rendu en français par les mots « et le travail qu'il nécessite 》 à l'alinéa g), et, à l'alinéa h), par « et toute besogne accessoire ».

Dans la recherche du concept énoncé on devrait normalement présumer que le choix par le législateur d'un terme plus précis manifeste sa volonté de restreindre la portée du terme employé dans l'autre langue, dans la mesure, bien sûr, où le terme plus général est susceptible d'être employé dans un sens aussi restreint que celui que suggère l'autre langue. Ceci sera d'autant plus vrai que dans un même texte ou encore mieux dans un même article d'une loi un terme est rendu en deux occasions de façon différente et d'une façon qui, non seulement les particularise mais aussi suggère une volonté de nuancer le concept lui-même. Force m'est de reconnaître cependant que la facture même de l'article 11 n'a rien de rassurant pour celui qui rechercherait via la seule sémantique l'intention du Parlement. Heureusement celle-ci se manifeste clairement à l'article 4 de la Loi sur le dimanche. Aussi, en interprétant les exceptions de l'article 11 on doit chercher à respecter la volonté de ne permettre le dimanche, sous réserve de l'exception contenue à l'article 4, que les travaux qui sont nécessaires à la bonne marche d'une société moderne.

La version française du paragraphe g) de l'article 11 me semble « assurer le mieux la réalisation de cet objet $\gg$ et, à mon avis, devrait être préférée à sa contrepartie anglaise ${ }^{6}$.

Autre exemple de divergence, l'arrêt Longtin c. Les industries Domco Limitée, où le juge Mayrand de la Cour d'appel analyse les deux versions de l'article 2261, paragraphe (3) du Code civil du Québec. Cet article se lit:

L'action se prescrit par deux ans dans les cas suivants :

$\dddot{3}$. Pour salaire des employés non réputés domestiques et dont l'engagement est pour une année ou plus.

en anglais : «For wages of workmen not reputed domestics and who are hired for a year or more. 》

Le juge Mayrand poursuit : «L'appelant soumet que ce qui lui est dû à titre de commission n'est pas un salaire et que de plus il n'est pas un employé selon le sens qu'il faut donner à ces mots dans l'article $2261{ }^{7}$. » L'appelant soutenait qu'il faisait un travail intellectuel et que cet article ne s'applique qu'aux travailleurs manuels. Il se fondait sur un jugement interprétant en ce sens la version anglaise de l'article. Le juge se reporte ensuite à Pierre Daviault et à Léon Guérin pour conclure que la version anglaise donne raison à l'appelant. * Il ressort de ces ouvrages que les mots \& wages of workmen... se rapportent à la rémunération d'un travail manuel et non pas à celle du gérant d'une grande entreprise commerciale ${ }^{8}$. Il poursuit ses recherches dans des dictionnaires de langue française et conclut que «les mots salaires des employés utilisés dans

6. B.P. Pétroles Limitée c. Sa Majesté la Reine, 1978, C.A. Montréal, no 10-000144-764,

7. Longtin c. Les industries Domco Limitée, 11 mai 1977, C.A. Montréal, no 09-000844-

761 , à la page 4 .

8. Id. à la p. 5 . 
la version française du même article n'ont pas une signification nécessairement aussi restreinte ${ }^{\ominus} \gg$. Et ce, principalement à cause de l'emploi dans la version anglaise du mot workmen. «L'appelant, un gérant d'entreprise, est un employé, mais il n'est pas un workman ${ }^{10}$.

Comme on le voit, les juges se font parfois linguistes, non sans habileté si l'on tient compte du fait que les linguistes eux-mêmes ne s'accordent pas toujours sur le sens des mots. D'ailleurs, ici, le traducteur ne s'était-il pas fait législateur?

Ce ne sont là que quelques exemples d'interprétation bilingue ; les pages de nos recueils judiciaires en comportent plusieurs autres. Pourtant, devant la grande concentration au Québec des jugements interprétant les deux versions d'un même texte, on est aisément tenté d'affirmer que si l'on excepte la Cour suprême et la Cour fédérale, seuls les tribunaux québécois accordent un poids égal aux versions française et anglaise des textes législatifs. Est-ce à dire que dans les cas où les versions d'un texte applicable à l'ensemble du pays divergent, les justiciables bénéficient de plus d'élasticité dans l'interprétation au Québec qu'ailleurs? Une interprétation qui sait être bilingue est parfois lourde de conséquences. Surtout lorsque, après avoir recherché l'intention du législateur sans la trouver, le juge recherche l'interprétation la plus favorable à l'accusé, en matière criminelle ou pénale.

C'est ainsi qu'en octobre 1977, la Cour d'appel du Québec, après avoir étudié les deux versions d'un traité d'extradition signé entre le Royaume-Uni et la France en 1896, se fondait sur la version française pour confirmer la libération de l'accusé, dans l'arrêt Le procureur général du Canada c. Armand Mekies ${ }^{11}$. Dans sa décision, le juge Albert Mayrand cite l'arrêt britannique The King $v$ s The Governor of Brixton Prison, portant sur la demande de liberration d'un citoyen français, présentée en vertu de la version française de l'article 10 du traité. Les juges s'étaient en effet penchés sur le même traité. Ils y admettent que s'ils avaient considéré la version française du traité, leurs conclusions auraient pu être différentes. Mais l'un d'entre eux considère simplement la version française comme une mauvaise traduction et conclut :

We are, in my opinion, bound to take the English version of the treaty as being the authentic version which the Courts of this country must consider,... ${ }^{12}$

Un second juge ajoute :

We ought not, in my opinion, in construing the treaty to use any knowledge which we may have of the French language. If we had to construe an extradition treaty with a country with whose language we were not familiar, we could only look at the English version of the treaty. It is a mere acci-

9. Longtin c. Les industries Domco Limitée, op. cit., à la p. 5.

10. Id. à la p. 6.

11. Le procureur général du Canada c. Armand Mekies, 1977 C.A. Montréal, no 500000061.

12. The King vs The Governor of Brixton Prison, (1912) 3 K.B. 190, le juge Darling à la p. 196. 
dent that the members of the Court are more or less familiar with the French language... I think that we ought not to look at the French version ${ }^{13}$.

Rappelons-le, ces mots ont été écrits en Angleterre en 1912. Pourtant, le contexte canadien actuel est-il vraiment différent? Et si nos juges se disaient un jour \& it is a mere accident that we are more or less familiar with the English language » bien que la version anglaise soit officielle, assisterions-nous à un séparatisme jurisprudentiel complet, à l'exception des causes portées à la Cour suprême, et à deux évolutions parallèles d'un même droit fédéral applicable à l'ensemble du pays ? On peut le penser. $\AA$ tout le moins dans les cas où les deux versions divergent. Le juge Mayrand s'attarde lui à la version française et lui donne raison. L'accusé aurait-il pu bénéficier de la même interprétation à l'extérieur du Québec ou bien lui aurait-on opposé les mêmes raisons que dans l'arrêt Brixton Prison ? Car, outre les intéressants problèmes de traduction que ce type d'interprétation soulève, on est tenté d'extrapoler et de tirer des conclusions d'une autre nature : hormis certaines exceptions, à l'extérieur du Québec, les juges québécois seraient-ils seuls capables de s'attarder au sens des deux versions, de les comprendre également et de choisir celle qui est applicable?

Peu importe en cela de savoir quelle version l'emporte, les deux sont également officielles. C'est pour cette raison que le juge ne cherche habituellement pas à retracer l'origine linguistique d'une disposition législative. Ainsi, nous avons ici deux exemples : dans l'un, l'arrêt Domco, c'est la version anglaise d'un article du Code civil du Québec qui l'emporte, dans l'autre, l'arrêt B.P. Pétroles, c'est la version française d'une loi fédérale qui l'emporte. Pourtant, on considère généralement que le droit civil est d'inspiration française et que les lois fédérales sont à l'origine conçues en anglais. Si l'on ajoute à cela les nombreux emprunts entre nos deux systèmes juridiques, droit civil - common law, l'origine linguistique des textes n'apporterait finalement que bien peu de lumière à l'interprétation.

Au chapitre du bilinguisme juridique, notons que le juge en chef de la Cour supérieure du Québec, Jules Deschênes, reprochait récemment à ses collègues du Canada anglais leur ignorance de la doctrine et de la jurisprudence québécoises. Comme pour bien démontrer le désolant hermétisme des juristes du Canada anglais face aux décisions rendues au Québec, il intitulait son allocution On Legal Separatism in Canada ${ }^{14}$. Il y indique que les deux grandes traditions juridiques du Canada ne se sont en réalité jamais fondues en un droit fédéral unique. Si les juristes canadiens anglais ne s'en préoccupent guère, les juristes québécois en éprouvent eux un certain sentiment de frustration. Car le juriste québécois est par définition et même par obligation bilingue. On lui enseigne à pouvoir à tout le moins lire la jurisprudence et la doctrine unilingue anglaise qui s'applique à l'ensemble du pays : il doit pouvoir com-

13. The King vs The Governor of Brixton Prison, op. cit., le juge Channell à la p. 197.

14. On Legal Separatism in Canada, an address delivered by the Honourable Jules Deschênes, F.R.S.C., Chief Justice of the Superior Court of Québec at the Judges Night Dinner of the Toronto Lawyers Club, Toronto, le 9 janvier 1978. 
parer les versions anglaise et française des textes législatifs dont les deux versions sont officielles. Comme en bien d'autres domaines, la réciproque n'est malheureusement pas vraie. Les juges du Canada anglais ne considèrent pour ainsi dire jamais les décisions, dont certaines importantes, rendues au Québec. Comment s'en étonner du reste : comme le démontre le juge Deschênes, chiffres à l'appui, la jurisprudence québécoise n'est en général pas publiée dans les principaux rapports judiciaires des décisions relatives aux lois fédérales, faute d'une demande des intéressés en ce sens. Lorsqu'elle l'est, c'est fréquemment sous forme d'une traduction anglaise qui laisse souvent à désirer. Qu'adviendrait-il ici encore si les juristes québécois n'étaient plus bilingues? La solution réside-telle dans une traduction généralisée? Encore faudrait-il que le Canada anglais en fasse la demande.

\section{Le traducteur législateur}

Même si, comme nous l'avons vu, les juges interprètent la rédaction première et sa traduction comme un tout, sans chercher à identifier comme tels chacun de ces deux procédés, il n'en demeure pas moins qu'il y a en fait rédaction puis traduction. C'est ainsi que malgré une certaine forme de communication entre le rédacteur et le traducteur, il arrive que le traducteur légifère, à l'insu du rédacteur. Par conséquent, il arrive aussi que le texte traduit soit préféré au texte d'origine, une fois soumis à l'interprétation des tribunaux, parce qu'il répond mieux à l'intention du législateur. Les exemples précités démontrent, entre autres, que l'intention du législateur demeure souvent fort obscure et devient souvent, en fait, ce que le juge croit être l'intention du législateur, à la lumière des règles d'interprétation existantes et du contexte qui lui est soumis.

Évidemment, l'unilinguisme législatif élimine ce genre de problèmes, mais il demeure une pure question d'orientation politique. C'est ainsi qu'à la suite de choix politiques, le gouvernement fédéral promulgue des lois officiellement bilingues, que ce bilinguisme législatif prévaut aussi au Québec, à moins que la Cour d'appel n'en décide autrement dans un proche avenir et que le reste du Canada demeure majoritairement unilingue. En ce qui concerne la rédaction elle-même, le législateur fédéral rédige presque exclusivement en anglais puis traduit vers le français ; le Québec rédige, depuis quelques années surtout, presque exclusivement en français puis traduit vers l'anglais. Les autres provinces rédigent en anglais et ne traduisent pas vers le français, à quelque exceptions près, notamment le Nouveau-Brunswick.

\section{Et vint la Charte de la langue française...}

A l'heure actuelle, seul le gouvernement du Québec manifeste l'intention bien arrêtée de changer cette situation avec l'adoption de la loi 101, dont le chapitre III entend régir la langue de la législation et de la justice ${ }^{15}$. Deux articles concernent particulièrement l'interprétation judiciaire ; ce sont l'article neuf et l'article treize :

15. Charte de la langue française, 1977, L.Q. c. 5. 
Art. 9

Seul le texte français des lois et des règlements est officiel.

Art. 13

Les jugements rendus au Québec par les tribunaux et les organismes exerçant des fonctions judiciaires ou quasi-judiciaires doivent être rédigés en français ou être accompagnés d'une version française dûment authentifiée. Seule la version française du jugement est officielle.

Ces deux dispositions auraient pour effet, si elles étaient déclarées constitutionnelles, non pas de bannir l'anglais, mais plutôt de faire du français la seule version officielle. Logiquement, dans le cas des lois et des règlements, cela ne soulève aucun problème démesurément grave. Bien entendu, plusieurs textes législatifs québécois en vigueur demeurent à l'origine anglais ou d'inspiration anglaise, et cette disposition rendrait seule officielle et donc applicable la traduction ou la version française de ces dispositions. Notons dès maintenant que l'article neuf rendrait impossible aujourd'hui la décision du juge Mayrand, déjà mentionnée, dans laquelle la version anglaise de l'article 2261 du Code civil l'emporte. Pourtant, étant donné la nouvelle volonté du législateur québécois, il n'aurait pu attendre que toutes les dispositions législatives anglo-saxonnes à l'origine ne soient plus en vigueur. La période transitoire aurait été fort longue. Le Québec est de toute manière noyé dans une mer juridique anglo-saxonne et continuera certes d'y puiser largement, tout en adoptant ses emprunts à ses besoins particuliers et donc à la langue française. Importe-t-il vraiment en définitive qu'en certains cas de plus en plus rares seule une traduction soit officielle, si éventuellement tous les textes législatifs québécois sont véritablement conçus dans un langage fidèle à l'esprit de la langue française ?

L'article treize pose des problèmes différents. Si les lois sont aujourd'hui rédigées en français, il en va différemment des décisions des tribunaux. En effet le Québec compte encore un nombre relativement élevé de juges anglophones. La plupart, surtout à la Cour supérieure, rédigent leurs décisions dans la langue des parties. La Cour d'appel surtout fait exception à cette règle. Les juges de cette Cour rédigent en effet dans leur langue maternelle, quelle que soit la langue des parties. Il pourrait difficilement en être autrement, bien que ces juges soient eux aussi bilingues, vu peut-être le haut degré de précision du langage juridique nécessaire à l'exercice de la justice en cette instance supérieure. Ce degré de précision est beaucoup mieux atteint avec l'emploi d'une langue maternelle. Les décisions rédigées en anglais ne sont en général pas traduites en français et le justiciable n'est pas toujours en mesure de les comprendre, surtout les décisions que la Cour d'appel rend en anglais.

Le Québec a-t-il tous pouvoirs sur la langue de sa législation et sur la langue des tribunaux? C'est précisément là la question qui fait actuellement l'objet d'un litige devant la Cour d'appel du Québec, à la suite de la décision du juge Deschênes par laquelle il avait déclaré inconstitutionnel le chapitre III de la Charte de la langue française. Advenant que le chapitre III et donc l'article 13 soient déclarés constitutionnels, les juges anglophones nommés par le fédéral pourraient se voir contraints de toujours rendre leurs décisions en français sous peine de voir seule officielle une traduction de ces décisions. Tous 
sont bilingues pourtant, la lecture des rapports judiciaires le prouve hors de tout doute, mais de là à rédiger en français, surtout à la Cour d'appel! Certes, les décisions de nos tribunaux devraient à tout le moins être accompagnées d'une version française, mais est-il bien souhaitable qu'une traduction soit seule officielle? Cette pensée fait presque frémir d'horreur qui connaît les grandeurs et misères de la traduction, les misères surtout. Notamment si l'on se souvient de l'arrêt Tamarak Construction Inc. vs The United Services Club Ltd. ${ }^{16}$, dans lequel le juge Casey de la Cour d'appel avait écrit, pour indiquer que l'une des parties avait changé d'avis, qu'elle avait eu a a change of heart ». Dans le rapport, on lit en français que cette partie avait eu une "transplantation cardiaque »... Puisque le Québec ne peut obliger le gouvernement fédéral à ne nommer chez lui que des juges francophones, lui faut-il vraiment sacrifier ainsi la rigueur de l'expression juridique? En attendant des jours meilleurs, il serait peut-être possible de limiter les torts éventuels en confiant aux juges la responsabilité complémentaire de revoir et d'approuver les traductions, à l'exemple de la Cour suprême.

Ainsi donc, le législateur fédéral se trouverait-il seul ${ }^{17}$ perpétuellement voué au bilinguisme législatif? Il représente aujourd'hui le seul niveau de gouvernement où les mécanismes nécessaires à la réalisation de cette politique soient véritablement en place. Ces divers mécanismes ne fonctionnent pas sans heurts évidemment mais ils fonctionnent. Les textes législatifs y sont tous traduits ainsi que les décisions de la Cour suprême et de la Cour fédérale. Or, il est permis de se demander s'il serait vraiment souhaitable que toutes les provinces prennent exemple sur lui et se lancent dans la traduction législative et jurisprudentielle de masse pour contrer le séparatisme juridique dans lequel le Canada s'enlise. En effet les francophones se plaignent sans cesse davantage de se voir offrir du fédéral tant de traductions ; ils réclament des textes de lois véritablement adaptés à leur langue, dès la rédaction.

Il devient évident que les modes de rédaction propres au common law ne les satisfont plus. On songe déjà à trouver un compromis entre les deux traditions juridiques, afin qu'il $y$ ait rédaction dans les deux langues et non plus uniquement rédaction anglaise puis traduction française. Les écueils sont pourtant nombreux et la solution idéale sera sans doute difficile à trouver puis à mettre en ouvre. Bien des changements seront d'abord nécessaires puisque le principe même d'une rédaction bilingue ne fait pas encore l'objet d'un consensus. Restent à résoudre les problèmes de mentalité, de main-d'œuvre et de volonté politique. La solution concrète est à l'étude et n'est guère encore qu'entrevue.

MARIE LAJOIE

16. 1971, C.A. 334

17. A l'exception peut-être du Nouveau-Brunswick, dont la Loi sur les langues officielles accorde une autorité égale aux deux versions des lois lors de leur interprétation. Loi sur les langues officielles du Nouveau-Brunswick, L.R.N.-B., 1973, c. 0-1, a. 14. 